\title{
The Importance of Accessible Information in Promoting the Inclusion of People with an Intellectual Disability
}

\author{
Melody M. Terras ${ }^{1, *}$, Dominic Jarrett ${ }^{2}$ and Sharon A. McGregor ${ }^{3}$ (D) \\ 1 School of Education and Social Sciences, University of the West of Scotland, Paisley PA1 2BE, UK \\ 2 North Ayrshire Health and Social Care Partnership, Irvine KA12 8EE, UK; Dominic.Jarrett@aapct.scot.nhs.uk \\ 3 Faculty of Humanities and Social Sciences, University of Strathclyde, Glasgow G4 0LG, UK; \\ s.a.mcgregor@strath.ac.uk \\ * Correspondence: melody.terras@uws.ac.uk
}

Citation: Terras, M.M.; Jarrett, D.; McGregor, S.A. The Importance of Accessible Information in Promoting the Inclusion of People with an Intellectual Disability. Disabilities 2021, 1, 132-150. https://doi.org/ 10.3390/disabilities 1030011

Academic Editors: Janet Finlayson and Stuart Todd

Received: 29 April 2021

Accepted: 21 June 2021

Published: 29 June 2021

Publisher's Note: MDPI stays neutral with regard to jurisdictional claims in published maps and institutional affiliations.

Copyright: (c) 2021 by the authors. Licensee MDPI, Basel, Switzerland. This article is an open access article distributed under the terms and conditions of the Creative Commons Attribution (CC BY) license (https:// creativecommons.org/licenses/by/ $4.0 /)$.

\begin{abstract}
People with an intellectual disability experience high levels of social exclusion and a range of health inequalities. Increased inclusion and participation in healthcare decision making have been identified as key to promoting inclusion in health and social care. However, achieving these objectives requires increased recognition of the communication and health literacy needs of individuals with an intellectual disability and consideration of the appropriateness of the information currently provided. In this article, we report the results of a pre-COVID-19 qualitative study exploring the provision and use of accessible information to support the healthcare inclusion of individuals with an intellectual disability. A total of 35 clinicians participated in focus group discussions, and 10 people with intellectual disabilities and 10 carers were interviewed regarding their experiences of using accessible health-related information. Qualitative data analysis using a framework approach highlighted the crucial role of communication partners when using accessible information, deficits in current National Health Service (NHS: UK's publicly funded health care system) information provision in the UK, and possible broader relevance of accessible resources. The implications of these findings for the provision of accessible information in a post-COVID-19 environment are explored, and recommendations are made for the increased integration and theory-driven research to inform the provision, content, and use of health-related information, especially the provision of online information, in the future.
\end{abstract}

Keywords: intellectual disabilities; person-centred; accessible information; inclusion; easy read; comprehension; psychology; cognitive load

\section{Introduction}

The national experience of COVID-19 has brought into sharp focus the continuing inequalities faced by people with intellectual disabilities. Numerous sources of data both nationally and internationally have highlighted its excessive impact on this segment of the population including excess deaths far beyond the level experienced proportionally in the general population [1-4]. Critical observation illustrates that this impact does not represent a new phenomenon: rather, it is a reflection and exacerbation of the existing differential health experiences of people with intellectual disabilities [5,6]. Coping with the impact of COVID-19 in terms of lockdown and increased social isolation also intensified the existing challenges experienced by people with intellectual disabilities and those who support them. Within survey work undertaken by the Scottish Commission for Learning Disability, some respondents with intellectual disabilities highlighted a lack of available, understandable information, while a greater proportion of carers also highlighted a lack of clear information, with regard to issues such as available support, PPE, and interpretation of restrictions [7]. A recent article (2020) from the University of Birmingham also highlights the need for accessible information during and beyond the pandemic, noting the often 'legalistic' tone of COVID-19 guidance and the complex language this can involve [8]. 
The lack of information clarity and access to understandable information is a longstanding issue for people with an intellectual disability. Addressing these issues is frequently identified as a priority within national and international legislation and strategy $[9,10]$ as part of a drive to achieve greater inclusion, self-determination, and improving the wellbeing of individuals with an intellectual disability. The Accessible Information Standard [11] implemented within the National Health Service (NHS) in England highlights 'better support to make lifestyle choices' (p. 50) as one of the benefits of the standard and its promotion of better information. Similarly, the United Nations Convention on the Rights of Persons with Disabilities (UNCRPD) sets out the provision of clear information in appropriate formats as a key part of promoting independence. The most recent report of the Chief Medical Officer for Scotland (NHS Scotland, 2021) [12] also sets out a clear imperative for involvement and accessibility:

Serious harm can result if we don't listen to the people we care for, and if they are not given the information and support they need to make informed decisions about their care... As we continue to encourage supported self-management, I ask that you consider whether there is more you can do in your daily practice to make health information and services more accessible to the people we care for. (p. 17)

To be able to participate to the extent that they choose in healthcare (and other) decision making, people need information to be made available to them in an appropriate format. This agenda is commonly labelled 'accessible information' (AI) and was defined within a position paper from the Royal College of Speech and Language Therapists in 2010 [13], as follows:

A supportive process of making information easier for people with learning [intellectual] disabilities, that firstly involves simplifying the linguistic message and secondly conveying the simplified message in different mode(s) of communication, i.e., not just the written word or spoken message. (RCSLT, 2010, p. 26)

A key element of this definition is the recognition of the complex and multifaceted nature of information provision. It highlights the importance of considering not only the content and format of the information provided, but also how this information is used and how its use is supported. From this perspective the provision of accessible information has two facets: (1) a content/format aspect of the information resource itself, and (2) a process aspect concerning its use, i.e., the dialogue that accompanies the provision of assistance to aid the understanding of the information provided. In explicitly recognising this process element of AI within their definition, the RCSLT acknowledges the opportunity to view AI as a broad agenda, encompassing not only the information product, but also the culture and practice elements that enhance its use and success (e.g., appropriate appointment lengths, person-centred practice, and awareness of communication preferences).

Despite this emphasis on process, the response of organisations and services to requirements for providing accessible information, such as the Accessible Information Standard [11], overwhelmingly focuses on the production of the resource itself. Frequently, these resources are text-based materials using simple language accompanied by images of some sort (e.g., symbols, drawings, or photographs), and are referred to as 'easy read'. While the development and promotion of such resources has proliferated in recent decades, significant questions exist regarding the actual benefits of the easy read materials currently being produced. As Sutherland and Isherwood [14] noted in their systematic review of evidence regarding easy read, while their benefits are undisputed by health professionals, the empirical evidence supporting their use is far from conclusive and remains in significant need of progression. A number of potential issues with easy read have been noted, including:

- The possibility that using images alongside text creates a greater cognitive load, rather than making comprehension easier [15].

- The possibility that simplification of text risks losing necessary detail and creating misunderstanding [16]. 
- The considerable scope for images to be interpreted in a variety of ways [14].

- The potential for limited awareness and/or accessibility of available easy-read resources within organisations [17].

Although a variety of guidelines exist that seek to embed consistency and quality in the production of easy read materials, these guidelines themselves carry inconsistencies, as well as frequently being devoid of any reference to a clear evidence base [14]. In light of these issues, the emergence of a critical discourse regarding information provision in recent years has been a welcome development, with authors such as Buell [18], Chin [19], Mander [20], and Oldreive and Waight [21], bringing much needed technical and theoretical insight to the issue, while continuing to recognise the validity of the values-based arguments supporting its implementation and addressing the persistent inequalities faced by people with intellectual disability.

While the experience of the COVID-19 pandemic has brought a dramatic new perspective to the longstanding challenges familiar to people with an intellectual disability (e.g., isolation and lack of access to necessary services), it has also starkly highlighted the essential role of clear, accessible messaging in public life. A report by the Scottish Health and Equalities Committee (2020) [5] regarding the human rights impact of COVID-19 noted the concerns of "Disability groups, human rights campaigners, minority ethnic representative organisations and those who provide advocacy services" (p. 22) regarding the need for the Scottish Government to improve its approach to inclusive and accessible information, in order to support informed decision making and reduce exclusion. This has been reflected in the concerns of people with intellectual disability themselves: the previously cited survey work undertaken by SCLD (2020) [7] highlights concerns regarding accessibility of information among respondents, with one stating that they were looking for, "Something simple to help me understand" (p. 8).

The COVID-19 pandemic has highlighted the importance of clear communication and messaging from governments across the globe [22] and specific advice to reduce the use of technical language in public messaging [18]. While the AI agenda encompasses far more than just these issues, it has much to offer at a time when collaboration between disparate partners in the common good has become a familiar phenomenon. Moreover, linking AI to the communication agenda surrounding COVID-19, and the analysis of its consequences offers a new route for continuing its evolution and reinforcing its relevance.

It is in this context that the study described here is presented. If the provision of accessible information is to achieve its aims, within a public health and individual context, it is essential to understand how to maximise the effectiveness of existing information resources that deliver health information in a way that supports individual communication and understanding needs. The qualitative research reported here was part of a larger mixed method research project exploring the provision of accessible information [23,24]. The quantitative survey element was used to assess the scope and scale of accessible information provision, with qualitative methods used to explore how accessible information is used.

The four research objectives were to:

1. Explore perceptions of the nature and range of information currently provided in terms of its accessibility.

2. Identify what people with an intellectual disability, their carers, and healthcare staff perceive as the important aspects of accessible information.

3. Explore how people with an intellectual disability, their carers, and healthcare staff make use of information to make it accessible.

4. Explore whether existing information is accessible enough to meet the needs of people with an intellectual disability, their carers, and health care staff.

\section{Experimental Section}

The research took place within an NHS Board area in Scotland. Prior to recruitment, ethical approval was received from the NHS ethics committee (West of Scotland Research Ethics Committee), along with local Research and Development Management approval. 


\subsection{Participants and Recruitment}

The research employed a qualitative approach to engage with people with an intellectual disability active to local services, carers (both paid and unpaid), and staff from a range of local healthcare services. Focus groups were used to gather data from staff, and interviews were used with individuals with intellectual disability and carers.

An overall breakdown of participants is shown in Table 1.

Table 1. Summary of participants.

\begin{tabular}{cc}
\hline \multirow{2}{*}{ Interviews $n=20$} & Individuals with an intellectual disability: 10 \\
\cline { 2 - 2 } 5 Focus Groups $n=35$ & Carers: 5 paid carers, 5 family carers \\
\cline { 2 - 2 } & Primary care: 5 \\
\hline
\end{tabular}

\subsection{Recruitment of Participants with an Intellectual Disability}

Participants (aged 18 years and over) were randomly selected from the active caseloads of the 3 local community intellectual disability teams. Individuals were excluded at this point if members of the community team identified them as inappropriate for interview at that time, for clinical, life circumstance, or risk reasons. The included participants had the project introduced to them by a familiar clinician, using an information sheet designed similar to the traditional 'easy read' standards. They were then contacted three days later, and a date was arranged to meet the researcher and subsequently for the interview to take place (if they chose to participate). The participants were given the option to be supported within interviews by a familiar individual, such as a member of learning disability staff, carer, or family member.

\subsection{Recruitment of Carers}

An unpaid carer was defined as an adult who looks after a family member, partner, or friend who needs help because of their disability. A paid carer was defined as an adult who was employed by an agency to provide care [25].

Carers were randomly selected from amongst those linked to the community teams' active caseload. Recognising that paid and unpaid carers could potentially bring different perspectives, five of each were recruited to the project: after being contacted by a familiar clinician, paid carers were contacted directly by the researcher, while interviews with unpaid carers were generally organised by the intermediary clinician.

\subsection{Recruitment of Healthcare Staff}

Staff from a range of services were invited to participate in the project: Learning Disability, Primary Care, and Mental Health services.

Past evidence has highlighted shortcomings in mainstream service provision to people with an intellectual disability, with Primary Care being a critical element of healthcare, demanding particular attention [26]. Information clarity is also critical for mental health services to support individuals effectively, where individuals may be displaying impaired or disordered cognition, as well as the potential for individuals to have an intellectual disability. Both Primary Care and mainstream mental health services should ideally reflect the aspiration that people with intellectual disability should be able to benefit from mainstream support as far as possible, in the same way as other populations. By exploring the use of AI across a range of services, there is the potential to create synergies of practice, which could benefit not only people with intellectual disabilities, but potentially others. No Adult Mental Health staff were able to participate in focus group discussions, but staff from Child and Adolescent Health (CAMHS) were involved via the researcher approaching CAMHS team leaders, and at their invitation, identifying one team meeting at which 
the focus group discussion could be carried out. Purposive sampling was used to target Primary Care staff (practice nurses, health assistants, health visitors, and district nurses) working within two specific GP practices from each locality-the practice with the most patients known to have an intellectual disability and the one with the least, as identified within the information held by the Learning Disability Primary/Acute Liaison Nurses. When a practice could not participate, the researcher contacted the next practice on the list, i.e., the next smallest or next largest.

\subsection{Materials and Analysis}

Three schedules were prepared (individuals with an intellectual disability, carers, and staff). A semi-structured schedule of questions was used to ensure consistency in areas probed in the discussions, covering four main areas: (1) communication, (2) easy-read information, (3) what makes information accessible, and (4) the broader organisational context of information provision. Examples of 'easy read' and standardised healthcare information (e.g., appointment letters) were used to help focus discussions within interviews with clients and carers.

The interviews and focus groups were transcribed verbatim and thematically analysed in a manner informed by the Framework Approach [27]. This approach supports the examination of a priori questions such as preferences and use of accessible information, as well as the identification of emergent themes [20]. Individual units of significance were identified within transcripts before being labelled; these labels were then grouped thematically and related to specific project aims. Initial categorisation of data was undertaken by the project researcher, then reviewed in partnership with two senior researchers within the team.

\section{Results}

\subsection{Interviews with Participants with an Intellectual Disability}

Three main themes emerged from client interviews:

- Experiences and preferences of health information;

- Support for involvement in healthcare;

- Perceptions of written health information.

\subsection{Experiences and Preferences of Health Information}

Participants with an intellectual disability (PID) spoke about the range of formats they receive information in, with verbal information being the most frequent, followed by 'easy read'. Audio was the least frequently experienced format. Of note is the fact that one participants with an intellectual disability had received information on bowel screening in multiple formats, i.e., easy read, DVD, and presentation, and found the presentation to be most helpful, noting that:

"it was easier when they showed you the things how it happened" (PID)

Participants' responses clearly indicated the importance of providing information in a format that works for the person, and at a level appropriate to their understanding. The challenge of pitching material at the right level was highlighted by one participant's description of a resource they had been shown as 'childish'.

\subsection{Involvement in Healthcare}

All participants indicated that they received support in relation to their healthcare, the main areas discussed being making appointments, dealing with appointment letters, and attending appointments. Most interviewees described receiving support to understand appointment letters, especially standard letters. Font size was identified by one as a barrier that prevented their reading appointment letters, while another described an element of avoidance with respect to letters about their health: 
"Usually when a hospital appointment comes in, I go 'here you go, there you go' [give it to spouse]. Cos I just don't like getting hospital appointments in ... And if I don't understand them [letters], I get my [spouse] to explain it to me." (PID)

Having support within appointments can be crucial to help health information be more accessible to people with an intellectual disability. Support was described by the participants as coming from a variety of sources, including family, advocates, and learning disability nurses. Encouragingly, one participant spoke about health staff speaking directly to them (as opposed to just their support person), which resulted in them having the confidence to ask for help themselves if there was anything they did not understand, even when a family member was present to support them. This further emphasized the importance of involving people with an intellectual disability in their own healthcare and providing an inclusive space that helps builds confidence, adding a further element to what makes information accessible.

\subsection{Perceptions of NHS Written Information}

Participants with an intellectual disability were shown examples of NHS written information within the interview and asked what they liked and disliked about them. The examples consisted of two appointment letters (a standard and easy-read version), two leaflets regarding confidentiality (a standard and easy-read version), a leaflet about the Learning Disability Service, and a booklet, entitled 'Beating the Blues' [28], about someone feeling down and how they could help themselves feel better. Easy-read resources were not preferred by all: with regard to the appointment letters, greater familiarity with the standard letter led some clients to state that they preferred it, while others highlighted benefits such as larger font size and the presence of photos of staff on the easy-read letter. The clients did not feel that all the 'accessible' aspects of the easy-read letter were necessary. Through discussion with one client who had problems with their eyesight, it became evident that it was the larger size of the font that was the most beneficial aspect: the pictures on the letter were not seen as helpful.

A clearer preference was seen in regard to the leaflets on confidentiality. Here, the standard format and its use of phrases relating to the use of data in line with legislation presented more issues for clients. Only one participant stated that they preferred the standard leaflet, specifying that they liked the fact it did not have pictures. Another client with poor vision, who stated a preference for the easy-read version, also indicated a preference for it not to have pictures:

"I think I prefer the big one [refers easy-read because it has bigger writing]. I've only got one eye, I'm blind in that eye. I would nae have pictures on it. As long as it gets to the point." (PID)

Responses to the Learning Disability Service information leaflet similarly demonstrated the variable benefits of symbols. The leaflet was written to easy-read standards and included content such as brief descriptions of professions accessible within the team, accompanied by a symbol representing them. A participant with sight problems again found the symbols to be of little benefit. By contrast, another client indicated that, while they could read some of the leaflet, they struggled with other parts and felt that the pictures helped in their understanding:

"I like the pictures cos it shows you that [points to picture of envelope] and you send a letter. That helps a lot." (PID)

However, the potential for symbols to be misinterpreted was starkly illustrated by one client who read the leaflet using the provided symbols, and interpreted the symbol used to represent Occupational Therapist (two hands reaching towards each other, depicted within a circle) as "Get married" (PID).

The equivocal response to pictures in the other materials contrasted with the participants' views on the 'Beating the Blues' resource. This employed a photo story format to 
explore an individual's experience of low mood, and how they managed it with advice from their friend (see Figure 1 below).

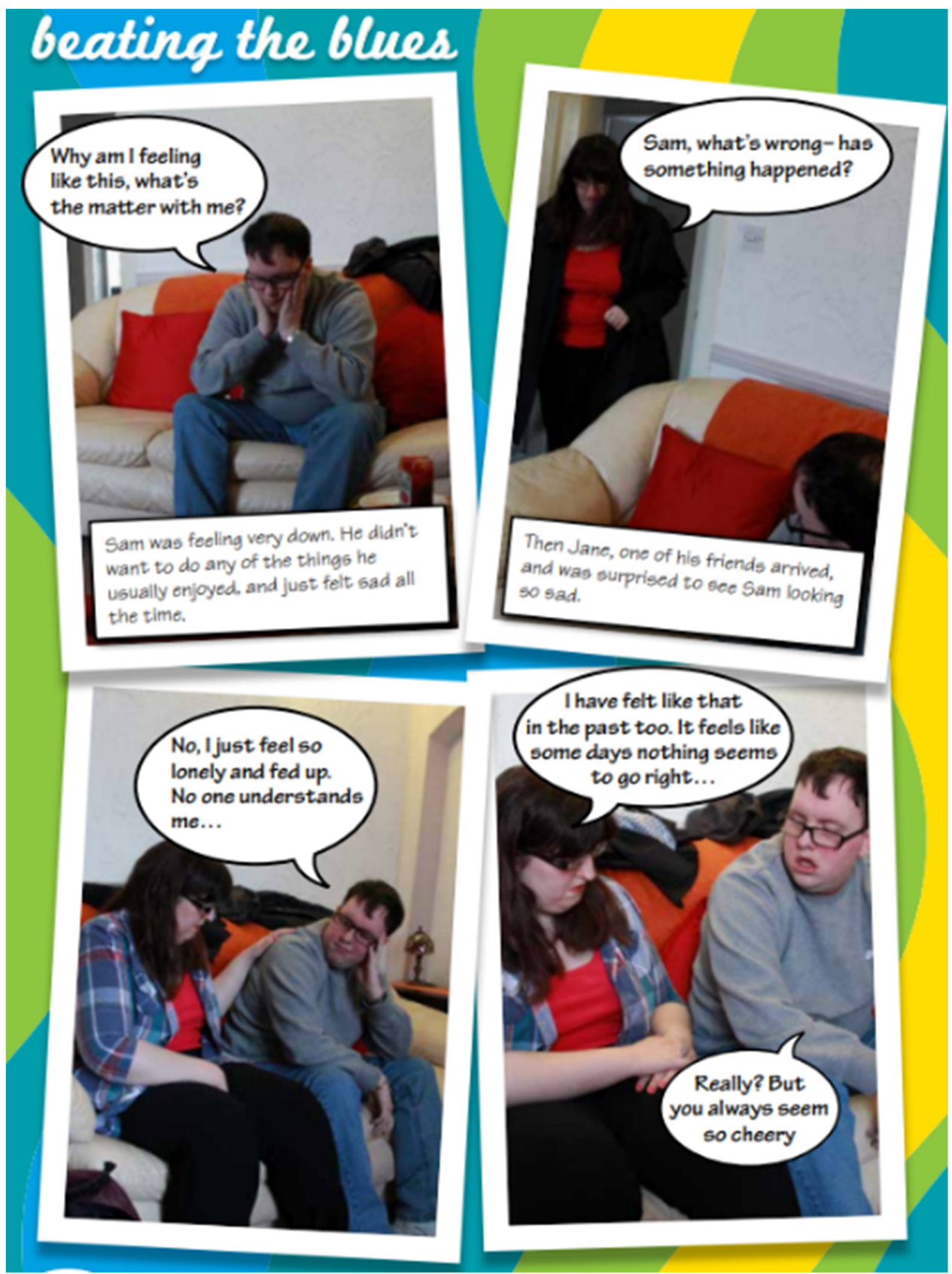

Figure 1. Extract from the 'Beating the Blues' resource. Copyright: NHS Ayrshire and Arran [28].

Of note is the fact that the one participant who indicated that they had not liked the pictures on all the other resources, did like the photos in this one. A number of participants commented on being able to relate to the individual with low mood:

"Oh that's me. I feel down. I feel so down and fed down and all that. You're right nobody understands me half the time [laughs] ... I understand how he-how he-how actually how he's feeling." (PID)

The colourful format and size of the pages (A4) was also remarked on positively. In addition, some clients, who indicated that they were unable to read the text, were still able to engage with the story as a result of the photo format. 
Although the participants with an intellectual disability expressed clear preferences concerning information resources, only two participants indicated that they had been involved in the production of easy-read materials, and one indicated that they had been involved in evaluating such resources. For one of the participants involved in production, this was an individualised resource regarding their life.

\subsection{Carer Interviews}

Five main themes were identified in the carer interviews:

- Carers as facilitators of clients' health and wellbeing;

- Carers' experiences of NHS services;

- Experiences of health information provision;

- Easy-read information: form and function;

- Perceptions of NHS written information.

\subsection{Carers as Facilitators of Clients' Health and Wellbeing}

Carers played an important role in facilitating the health and wellbeing of the person they supported. As well as providing practical support, for example, to attend appointments, they were also critical in supporting communication processes, for example, understanding the need to take medication.

Variable literacy skills among supported individuals were highlighted, with some participants with an intellectual disability described as unable to read, while others, although they may be able to recognise words, had limited comprehension of their meaning. It was also noted that individuals with an intellectual disability would often focus on single aspects of information that they could recognise (e.g., their name or a time) and neglect other content. One paid carer described someone they supported as having the ability to read, e.g., an easy-read confidentiality leaflet, but as lacking the confidence in themselves to be sure they had understood it:

"We read things to her and we say, 'well this means such and such', 'aye I did think that but I wasnae sure'. She just lacks really a lot of confidence in herself." (paid carer)

Reflecting their understanding of the person they support, some carers indicated that they would take on responsibility for some decision making, including taking the decision to opt out of healthcare, such as screening processes. Some unpaid carers described themselves as filtering out information that they did not deem relevant for their family member to be aware of:

"We monitor what we give them we don't give them too much but what we think they can cope with." (unpaid carer)

Supporting communication within the appointment also had a key role: both in terms of helping the individual to communicate what was wrong with them, and in attending to what was said by the clinician. Understanding the outcomes of an appointment was also an essential aspect:

"... if need be we will attend with them so that information they're receiving from the GP or the hospital is getting back to us because of sometimes, you know they don't retain that information." (paid carer)

Carers also discussed the role they played in informing the person they supported about their health and emphasised the importance of tailoring information to meet the needs of the individual. Two of the ways that were identified were (a) using simple language, "Oh I just explained it in simple terms [speaking about menstrual cycle problems]. No point in getting complicated about it. You know, just things that she would understand." (unpaid carer); or (b) picking out the key aspects of information: "It was booklets and booklets we were given but I also think that some of the information was that you had to read through it to pick up the main parts to be able to explain it" (unpaid carer). 


\subsection{Carers' Experience of NHS Services}

In terms of the experience of NHS services, the actions and attitudes of individual staff were identified as contributing to positive experiences:

... when we knew it was going into an adults ward, we thought, 'oh, how's this going to work? Somebody's got to be with him like aw the time', and they tain it onboard and we got a side room and they got me a bed and I stayed like, with him, and "spouse* came and we done it in like kinda shifts, back and forward. (unpaid carer)

However, they were also identified as contributing to negative ones:

"... certainly when he attends appointments, it depends on the individual nurse or doctor and their own experiences or whatever and how they even interact with them. Sometimes you've got an appointment and they're awkward or they don't know how to interact." (paid carer)

Staff failing to communicate effectively with the individual, and electing to address the carer instead, was experienced by several interviewees. Inefficient information provision was also described (e.g., appointment letters in inaccessible formats), as was a failure to recognise carers as a valuable source of information. Positive system responses, which were highlighted as helping with engagement, included appointment reminders by phone instead of letter and access to specialist practitioners. Other potential accommodations described by carers align very much with good practice highlighted elsewhere, such as allowing longer appointment times and engaging with staff teams in advance to facilitate learning about the individual [29]. Clinicians' spending time with individuals to introduce themselves was also suggested as a possible improvement, this being couched within a recognition of the busy-ness of acute environments, and the challenges presented by shift changes. For example,

I know it's busy wards and that and I feel I understand her. But just sometimes a wee bit of time to sit and talk and let them get to know you, like [PID] get to know the nurse. And of course, staff changes every two or three shifts as well which does nae help but that is, that's a fact of life ... (paid carer)

\subsection{Carers' Views and Experiences of Health Information Provision}

Carers described receiving information in a variety of formats, predominantly verbally. Some paid carers described accessing DVD information resources through their organisation, although one carer suggested that DVDs may provide too much information and could be a source of anxiety for an individual. Experience of plays or dramas as information provision was limited. One interviewee recounted accompanying a group of individuals to a drama-based workshop on hate crime, which was found to be extremely successful in engaging those present, as well as having a lasting impact on them:

Very helpful [workshop on hate crime], em they went on about it for weeks and weeks. And one of the guys were like' are we going again next week to something else?' So they really, really took it in. Em, and it brought one of the women I was supporting out their shell, like, answering all the questions, not really needing much support she was just shouting out and giving all her answers. Which was really nice to see. (paid carer)

\subsection{Easy Read: Form and Function}

In terms of easy-read resources, the carers described its usage as limited within the NHS, with one speculating that this was possibly linked to the additional work required in developing easy-read materials. For example:

"I think it depends on the individual [referring to NHS staff's knowledge of easy-read]. If the individual's willing to do the work for it. Because it's no em, it takes longer than just saying right, can I get a standard letter written." (unpaid carer)

Aspects of easy read that were highlighted as beneficial were the reduction in jargon and use of simple language: 
... you can't misread them [easy-read information]. It's clear, it's, when you've got the picture of the clock then it tells you the time, so it's straightforward. Em, if you can sometimes feel, the normal letters, you've gone through a paragraph before you get to the actual appointment sometimes. (paid carer)

The incorporation of images was also discussed as positive, both in terms of supporting engagement and creating a more visually appealing resource. However, there was no clear preference as regards to the type of image: the important thing was that they were there, in some form.

The carers described a variety of ways in which they made use of easy-read materials. Some described using it as a support when explaining information. The way in which an easy-read material highlights key information and how it helps to structure a conversation were viewed as a major benefit:

"If you've got this big [topic] and you're like right, where do I start, where do I start explaining stuff, whereas the easy-read then-the information that the guys need to know is there and you're just passing on that information." (paid carer)

A number of carers described the way in which easy-read materials facilitated the involvement of the individual within a conversation, through presenting the information in a simpler and more engaging manner. One carer contrasted this with the challenge of involving their family member in a conversation regarding guardianship, unsupported by such materials:

... we didn't get anything like wee symbols or anything, cos the lawyer would nae do anything like that. But it's something that, if there was information out there, it makes it easier for somebody like the likes of [family member] to understand, then it makes the parent or carer's life a bit easier as well. (unpaid carer)

The potential of broadening out these benefits to a wider population were described by some: one interviewee spoke of its possible benefits for the elderly, while another spoke of it in the context of low literacy, or not having English as a first language. Alongside this, there was, however, a recognition of its challenges, including the breadth of needs across the whole population, and the potential for it to be viewed as childlike. The key point was to keep the information simple and jargon-free: "Cos it is sad to see that no everybody's able to read, even kinna like normal, kinna like no having like a disability. Em to have like some form that everybody can understand, like a picture form or just easy-read. Kind of, keeping it basic." (unpaid carer).

\subsection{Perceptions of NHS Written Information}

The carers had the opportunity to comment on the same appointment letters, confidentiality and team leaflets, and the 'Beating the Blues' resource, as were shared with clients. In terms of the appointment letter, there were clear benefits ascribed to the easy-read template in terms of involving clients, as well as the practical role it could serve as a clear visible prompt when, e.g., displayed within the home. For example, "There's no as much jargon there [benefits of easy-read], it's got basic, straight to the point, what they need to know, so it's less to like you know, take in for her to try to em, try to understand" (paid carer).

Some carers expressed a preference for the standard template for themselves, while others recognised that even the easy-read template would fail to engage those with more complex needs. With regards to the confidentiality leaflets, aesthetic and structural elements of the standard leaflet (brighter colours, better use of sub-headings) were viewed as positive by some, while the illustrations and brevity in the easy-read version were viewed as positive by others. In regard to the latter, the concern was expressed that while the easy-read version was helpful, it was also open to misinterpretation. The service information leaflet was generally well received by carers, but with a realistic appreciation of its limits, along with some suggestions for improvement (including larger and more appropriate symbols). The 'Beating the Blues' resource, to which participants with an intellectual disability responded positively, was also viewed positively by carers. Similar to the participants with an intellectual disability, the use of photographs was broadly seen as positive, with one 
carert commenting on the benefits of the protagonists' expressions being visible. However, concerns regarding it were also expressed:

Right I've got two opinions here [about Beating the Blues booklet]. The first one, it's amazing! For a young person who's not visually impaired or autistic. An autistic child that would drive them crazy. Because one it's too bright, it's too busy and it's too cluttered. (unpaid carer)

In terms of the production of accessible materials, a minority of carers described experiences in this regard. Two paid carers spoke about client involvement in the production of easy-read materials used by the organisation, while an unpaid carer spoke about generating social stories for their family member in order to describe activities such as taking the bus to an appointment.

\subsection{Staff Focus Groups}

The staff focus groups had a broad focus, touching on general issues regarding communication and accessible information, as well as their broader organisational context.

Six themes emerged from across the focus group discussions:

- Experiences of current health information provision;

- Importance of accessible information;

- $\quad$ Easy-read: form and function;

- Making information accessible: processes and challenges;

- The role of carers in providing support to convey health information;

- Applicability and future of accessible information.

\subsection{Experiences of Current Health Information Provision}

The participants identified a wide range of mediums being used, including verbal discussion, easy-read materials, DVDs, apps, and other internet-based resources; although in relation to the latter, accuracy of information was highlighted as a concern. Current information provision was perceived as rather service-centric, not always aligned with the literacy needs of the client group and often framed within the traditional passive tradition concerning patient expectations of clinicians. For example: "There's an assumption that everyone attending an NHS appointment is literate, it's not just our service, it's every appointment that's offered" (CAMHS Participant).

\subsection{Importance of Accessible Information}

In general, the increased provision of information in accessible formats was welcomed by staff and viewed as necessary to help address a number of practical barriers to healthcare. Staff perceived standard information provided in less accessible formats as leading to missed appointments and patients being unprepared for procedures. Concern was also expressed by staff over the emotional aspects of health-related communications. Healthrelated communications could be a source of anxiety, and accessible information may ease the emotional and practical difficulties experienced by some:

"I know what you're saying but for the sake of those people who wouldn't like it [easy-read materials], if it got the people who are intimidated or are unable to read it or are scared of letters that come in, if it's something that is far more friendlier." (CAMHS participant)

\subsection{Easy Read: Form and Function}

The staff were most familiar with easy-read materials as a source of accessible information and discussion. Such materials were viewed as appealing to a wide range of client groups due to their general aesthetic appeal, use of everyday language rather than medical terms, and concise and focused nature. The use of some form of graphic within easy-read material was discussed in depth, with staff reflecting on how graphics in the form of drawings and photos were generally beneficial in facilitating engagement and understanding. Despite their usefulness, the staff recognised the complexities of selecting 
the appropriate image type for different types of information, and different client groups. For example,

"So for people with autism especially, I think if they see a picture they expect it to be pretty much how that picture is. Em, it depends on the service-user really and what their needs are, and how they interpret things. Because some people are very, extremely rigid." (LDS participant)

In terms of their use, the staff considered how easy-read resources were useful to structure and support communication about health-related topics. For example:

"I think that some of the Fair leaflets that we've used ... pictures, information and you can usually kind of structure what you want to say around they leaflets. So I've found them to be beneficial." (Primary Care participant)

"... it [easy-read] provides a framework to work from I suppose. I mean sometimes, you know, having that framework and then going through the things on the leaflet rather than you just going in and trying to remember everything. It's good to have a framework." (LDS participant)

\subsection{Making Information Accessible: Processes and Challenges}

The topic of engagement with information to make it more accessible was a major focus of discussion, with staff frequently identifying a range of factors that influenced this, including client characteristics (e.g., ability and communication) and the expectations of clinicians. The staff discussed, in detail, how they would personalise available information in order to tailor it to the individual's abilities and promote engagement, as well as developing individualised materials from scratch where necessary. For example, "I think if you make it personal ... then you know they're going to understand it because it's personal to them" (LDS participant).

With regards to the skills required to individually tailor information provision, the staff reflected on how this skill developed with experience and was informed by both their understanding of the individual and the client group in general. In order to make information accessible, the staff described how they would establish a baseline of comprehension for each client; choose an appropriate resource; tailor the information to the clients' needs; and check understanding. Once a suitable approach was identified, they aimed to maintain consistency. Working through and repeating information were identified as key elements of promoting understanding. For example,

They'll say they understand it and then you can tell at the end of the session that they're very doubtful they've actually understood most of what they've seen and they might need to go over that several times, before they actually give consent to attending the appointment. (LDS participant)

While recognising the many benefits that can be gained from AI provision, the staff also discussed the challenges they perceived to be associated with this process. Many challenges were practical in nature and reflected constraints imposed by the existing resources, available technology, and time.

Not often enough [how often they use easy-read] cos we don't have time to produce it. Because it takes time. So many times I think it might have been useful to, but that planning time is not available... when you sit in front of one of our computers and try to do something it takes three quarters of an hour... (CAMHS participant)

\subsection{The Role of Carers in Providing Support to Convey Health Information}

The staff discussed the important contribution made by carers in helping to provide additional context and convey more fully the health needs and the expectations they had concerning the carer's role. For example,

A lot of patients will tell you what they think you want to hear. They will nod and go along with, 'yes that's correct' ... it's not until you've maybe got a carer in the room at 
the time, ... to say that's not what they do, or that's not what it's like. So it is, it [carers] is a valuable source of information. (Primary Care participant)

"I think it's vital [the role carers in conveying health information to staff]. You've got to have that communication. If you're going to support a patient you need as much as you can get." (LDS participant)

The nature of the carer (paid or unpaid) also influenced staff practices concerning the use of accessible information resources. For example:

"I think you can be more direct with staff teams once eh, 'this, this is how this'll impact on the person's health'. With families, well you go a bit slower and just, plant a seed." (LDS participant)

"... If the person is totally dependent on the carers then they are sort of dependent on their [carer's] lifestyle. What they're used to, their circumstances. Finances comes into it as well, sometimes where they live, em and it can just be the norm for whatever their pattern of behaviour is." (LDS participant)

\subsection{The Wider Applicability and Future of Accessible Information}

The wider applicability of AI was also considered with respect to the general population and vulnerable groups (e.g., children, the elderly), and it was recognised that there were potential advantages to this. The difficulties inherent in a system designed around general needs were acknowledged:

I think that, in my head, the usefulness of it would be just em, for us to adapt to client needs rather than clients having to adapt our [service] needs. So if you're using something generalised you're asking everybody to adjust to what you're giving. But if you take a step back and adjust things then I think that's more client friendly in general. (CAMHS participant)

However, despite widespread appreciation of the advantages of easy-read format, the staff also expressed concerns about broadening its implementation, especially the resource implications:

"I don't think that's necessary for everyone. I think there are certain groups of patients that you would decide that I know what I can give you that you would understand. I think, number one, that would cost a lot of money to reprint everything that you've got into an easy-read situation." (Primary Care participant)

\section{Discussion}

The findings from the interviews and focus groups are a rich source of experiential data from individuals with an intellectual disability, staff, and carers concerning their use, expectations, and preferences for regarding information to support health and well-being.

Six important themes to inform future practice are evident:

- $\quad$ Easy-read as a resource to present health information in an accessible way;

- Accessible information is more than just easy-read;

- Accessible information as a process;

- The importance of using an AI approach to information provision;

- The challenges of using easy-read materials to convey health information;

- The relevance for accessible information in the context of the COVID-19 pandemic and beyond.

Carers and clinicians described using easy-read resources to structure health-related conversations and present information in an accessible way. The involvement of CAMHS and Primary Care participants in the focus group discussions provided a clear indication that they, along with the Learning Disability staff, recognised the importance of making information accessible within their services and could provide examples of their current practice of tailoring information and its presentation as needed. However, accessibility extends beyond easy-read materials; carers also appreciated the benefits of diverse and 
engaging formats, with one carer highlighting the positive outcomes of including a group of clients in a drama-based workshop on hate crime. Buell (2020) discusses the possible benefits of supplementing easy-read resources with other mechanisms such as role play or real-life experiences to support increased understanding [18]. Waight and Oldreive (2020) also note the potential of video and narrative formats as means of promoting understanding, encouraging the exploration of narrative (including drama sessions) to enhance health information provision [30].

Our findings highlight that these less didactic forms of information provision are appreciated by individuals with an intellectual disability and carers alike and could well have benefits through being inherently more linked to a social, discursive process within which relevant meaning can be constructed with an individual. For instance, the response of participants with an intellectual disability and carers to the 'Beating the Blues' resource is notable. While it very much evidenced the power of narrative approaches [30], it also reflects recommendations regarding a general preference for photographs as they are seen as being more respectful, and further illustrates the potential importance of information resources having a personal connection to the individual reading them (e.g., it uses their name or image [21]). It may be that it was this more personal connection that enabled the use of the 'Beating the Blues' resource to stimulate discussion and self-reflection. Such an interpretation is in keeping with the very welcome trend within the AI evidence base, which recognises the importance of the social construction of meaning and a reminder to consider the context in which the resource is used [18,31].

As outlined earlier, the provision of accessible information is a complex process involving both resource and process elements. Staff often described themselves as working in a way that very much aligned with Oldreive and Waight's (2013) description of an evaluative and reflective process around the use of $\mathrm{AI}$, including establishing a baseline of understanding, developing appropriate materials, reviewing their use, and then adjusting as appropriate [21]. The facets of easy read, which were identified as beneficial in this study, such as a tight focus on key information, align with the existing recommendations around its production, while also reflecting some of the contested areas emerging within recent years, such as issues concerning the inclusion and interpretation of images. For example, the larger font was especially valued by those with a visual impairment, more so than the inclusion of images [15]. Although images were appreciated by many, they could serve as a source of visual interference and ambiguity for others.

Our findings indicate that carers and staff appreciate the importance of delivering accessible information to individuals with an intellectual disability as part of a supportive, ongoing relationship, again highlighting the process aspect of information provision. The role of the 'information facilitator', described by carers and staff, and the process element of AI that this embodies, are critical components of information provision. A lack of confidence in their abilities was highlighted as a barrier to making use of written information in the client interviews, as well as in those with carers, further highlighting the way in which this facilitative relationship may be of benefit. As well as contributing to the construction of relevant meaning, supportive relationships may play an important role in enhancing an individual's belief in their own abilities and promote self-esteem, which is a key enabler of independence, self-determination, and inclusion [31]. While the social and supportive elements of constructing meaning were clearly evidenced in the responses of clients, carers, and staff, the way in which this was described by many participants in this study (e.g., filtering information) highlights concerns around the potential for exclusion, misinterpretation, or partial information, when supporting the use of information, generally, and easy-read documents, in particular. Some carers were taking a very active role in making decisions regarding healthcare; for example, one participant spoke of picking the elements of text to share. Mediation in understanding information in any format has the potential not only to improve knowledge and understanding but can potentially be detrimental to correct understanding and meaningfully informed decision making. Potential misunderstandings may be exacerbated by the easy-read resource, reflecting the 
at times 'cosmetic' nature of easy-read implementation and the superficial 'understanding' they support [17]. Therefore, it is essential to understand how information resources are being used and the opportunities and challenges they present.

The need to develop a detailed understanding of how resources are being used to convey information is highly salient, as the COVID-19 pandemic not only highlighted the importance of providing accessible information for individuals with an intellectual disability [32], but it also disrupted and altered many of the supportive mechanisms for its use. As we return to the 'new normal', this is an opportune time to rebuild process and practices to help maximise the potential of $\mathrm{AI}$ in order to make health information more accessible. Although lockdown impacted heavily on the well-being and the support networks of individuals with a learning disability and their carers [33], it also stimulated new ways of working, models of service delivery and the emergence of new connections, and the mobilisation of new networks within communities [34]. Developing (or re-establishing) those connections damaged by the experience of repeated lockdowns, and, with them, the facilitative environment for realising the process elements of AI, would benefit from being informed by the concept of 'unhurried conversations' [35]: careful and kind care, delivered at a pace that allows a trusting, meaningful, and productive relationship to emerge between the individual and care giver (likened by Fog Heen and Montori to a dance).

It may also open up scope for expanding the involvement of people with intellectual disability and others in resource production. Our findings indicate that participants had limited involvement in the production of resources. Such findings, although disappointing, are not unexpected as the limits of existing approaches to the co-production of resources are being recognised [36]. Increased involvement of not only individuals with an intellectual disability but also those who support them (staff and carers) has the potential to ensure that accessibility of information is maximised both in terms of the content of the resource and the process of use. In addition, through involving people with intellectual disabilities as equal contributors alongside other members of the general population, and through using technology and other new mechanisms for involvement, it may be possible, as Chin and Pelletier (2020) say, that "accessible information might escape the ID "ghetto"" (p. 334) [36]. This is all the more important given the prominence of digital approaches in the response to the pandemic. In terms of connecting people with services [37], for social reasons, and as a means of accessing information, technology has been important for all, but not equally available to all [38].

The evidence concerning the nature and practice concerning the provision of information are rapidly being established, and we propose that the future development of information provision would benefit from increased integration and multi-disciplinary working. In particular, we advocate the importance of considering the insights offered by a psychological perceptive to help inform the characterisation of the user, the design of the resource, and the process of use. The insights offered from a psychological perspective are already evident within the literature, for example, the consideration of psycholinguistic work concerning theories of reading to inform our understanding of the comprehension of easy-read, the increased emphasis on considering individual differences, especially cognitive factors that influence the processing and comprehension of information [18], and the use of Brofenbrenner's theory to contextualise the use of AI [23].

Increased consideration of a psychological perspective also offers insight into understanding the design of information in online contexts. The COVID-19 pandemic has accelerated the shift to digital, with online consultations and the provision and dissemination of information in an online/digital format becoming the new norm. The increasing delivery of healthcare and information in digital formats raises a number of significant challenges for individuals with intellectual disability who already experience high rates of digital exclusion and range of challenges concerning access, digital skills, and safety [39-42]. The increased use of online service delivery has consequences for the existing delivery and support systems concerning the provision of accessible information and may reduce the opportunities/alter the nature of the in-person support that is so valued by staff, carers, and 
individuals with intellectual disability alike, as demonstrated in the results of this study. The online provision of information may also exacerbate the well-recognised and previously discussed difficulties concerning the design and comprehension of health-related information. We propose that insight may be gained from the well-established evidence demonstrating the importance of considering cognitive load $[43,44]$ in the design and presentation of information resources. The impact of cognitive load has been extensively applied and evaluated in the design and provision of online educational resources [45], so it has immense potential to inform the provision of health-related accessible information. The key aim of the cognitive load theory is to inform the design and presentation of instructional material to avoid overload. It distinguishes between Intrinsic (the cognitive demands of the task itself) and Extrinsic load (processing demands out-with the task itself). Extrinsic load is generally produced by the format in which the information is presented, e.g., text: describe a shape; or image: show a picture of the shape; these can be manipulated and are ideally to be kept to a minimum. Therefore, information should be presented in way that minimises Extrinsic cognitive load in order to free up information processing resources to deal with the task at hand, i.e., understanding the information being presented.

Adopting a cognitive load framework allows the systematic exploration of many of the challenges previously discussed and is very much in keeping with the existing recommendations. For example, consideration of the differential impact of extrinsic and intrinsic cognitive load offers an explanation for the visual interference in the participants of this study concerning images, as it allows the detailed consideration of both the load imposed by the resource and the abilities of the user. It also supports the systematic exploration of the intrinsic and extrinsic influences concerning the use of easy-read and captures the influences concerning the role of cognitive effort and the importance of considering the skills and abilities of the user, as advocated by Buell et al. (2020) [18]. Research examining how cognitive load is distributed across participants in collaborative learning tasks [45] offers insight into the conflicting findings and difficulties experienced when supporting an AI resource, as the strategies/language used may increase the cognitive load of the task and be more of a hinderance than a help [18]. Lastly, the emphasis on instructional design, which underpins a cognitive load approach, ensures that the design and content of the resource, the profile of user, and how they may interact are considered from the outset. This discussion illustrates the integrative benefits that can be gained by synthesising existing findings and situating future research within a wider framework. Although psychologically informed frameworks have much to offer, as we outlined here, we appreciate and encourage the application of frameworks from other cognate and design areas to help integrate, develop, and inform the future provision of accessible information.

\section{Conclusions}

The experiential data reported here offers considerable insight into the views, expectations, and preferences of individuals with an intellectual disability, carers, and staff, concerning the use of accessible information to support well-being and inclusion and to reduce health inequalities, enriching the evidence base concerning accessible information. A major strength of this study was the sampling of a wide range of staff and the provision of concrete examples of a range of information resources, which helped to stimulate discussion and generated considerable insight into the sources of preferences and concerns. Similar to most of the existing evidence, this study was conducted pre-COVID-19, so we took the opportunity to not only discuss how the findings relate to current evidence, but also how they may inform future information provisions.

The COVID-19 pandemic has changed the landscape and accelerated the move to digital, and now is an ideal time to reflect on the current state of the art with respect to the provision of accessible information in a post-COVID-19 environment. Although the current evidence-base is developing as a result of vibrant commentaries, reviews, and rigorous experimental studies, there is a distinct lack of integration. Although key issues and themes are evident and there is increasing use of theoretical perspectives, the existing 
evidence-base is rather fragmented. We propose that greater integration can be achieved by increased multiple disciplinary working that allows the application of a wider range of theory and research. The provision of accessible information is highly complex as it has the potential to address the needs and preferences of a diverse audience; therefore, it is essential that we draw upon the expertise of disciplines who are highly experienced in these areas. To ensure the quality of AI provision, future research would benefit from being more theory driven, contextualized, and psychologically informed. As we discussed, psychological theory and research have much to offer not only to the design of the information resources, but they also offer immense potential to characterise the profile and abilities of the user, which is key to personalisation. That said, psychological insight is just one strand that we identified and discussed, and we endorse increased consideration of a wide range of disciplines and the frameworks they offer for integration in order to inform the future provision of AI.

The COVID-19 pandemic posed considerable challenges for all but particularly impacted individuals with intellectual disability. As described by the Mental Health Foundation [46], although we may have been in the same storm, we were not all in the same boat (Mental Health Foundation, 2020). Recognising the diversity of those experiences and ensuring that the experiences of people with intellectual disability are viewed as equally powerful and important as those of other marginalised groups will be a key step in ensuring that relevant shared learning emerges from the pandemic. In this respect, the developing critical discourse around accessible information provision has much to offer with regard to developing resilient supports delivered on a more equitable basis. However, potentially, the greatest value will stem from finding a way to connect that discourse to a broader exploration and change of service structure and delivery.

Author Contributions: All three authors have contributed towards this paper: Conceptualization, D.J. and M.M.T.; methodology, D.J., M.M.T. and S.A.M.; formal analysis, S.A.M., D.J. and M.M.T.; investigation, S.A.M., D.J. and M.M.T.; data curation, S.A.M., D.J. and M.M.T.; writing—original draft preparation, D.J. and M.M.T.; writing-review and editing-M.M.T., D.J. and S.A.M.; project administration, D.J. and M.M.T.; funding acquisition, D.J. and M.M.T. All authors have read and agreed to the published version of the manuscript.

Funding: This project was by funding from the Chief Scientist Office (Scotland) Priorities and Needs Programme: Social Determinants and Interventions for Health. Ref: 2012AA002.

Institutional Review Board Statement: The study was conducted according to the guidelines of the Declaration of Helsinki, and approved by West of Scotland Research Ethics Committee (ref: 12/WS/0003), 5/4/2012.

Informed Consent Statement: Informed consent was obtained from all subjects involved in the study in line with ethical approval.

Acknowledgments: First and foremost, the research team would like to thank all those individuals who participated in interviews, focus groups, and surveys as part of this study, the services who facilitated this involvement, and those who helped to shape the various resources used within the project. Thanks are also extended to the various organisations who met with us as part of the background research to this project, including Sense Scotland and the Scottish Consortium for Learning Disabilities (SCLD). This project was greatly enriched by having a wide range of experiences and perspectives reflected within the Steering Group, and we remain very grateful to all those who participated in it.

Conflicts of Interest: The authors declare no conflict of interest. The funders had no role in the design of the study; in the collection, analyses, or interpretation of data; in the writing of the manuscript, or in the decision to publish the results. 


\section{References}

1. Scottish Government. COVID-19 and Disabled People in Scotland-Health, Social and Economic Harms; Scottish Government: Edinburgh, UK, 2021.

2. Public Health England. Deaths of People Identified as Having Learning Disabilities with COVID-19 in England in the Spring of 2020; Public Health England: London, UK, 2020.

3. Gleason, J.; Ross, W.; Fossi, A.; Blonsky, H.; Tobias, J.; Stephens, M. The Devastating Impact of Covid-19 on Individuals with Intellectual Disabilities in the United States. NEJM Catal. Innov. Care Deliv. 2021. [CrossRef]

4. Fair Health; West Health Institute; Makary, M. Risk Factors for COVID-19 Mortality among Privately Insured Patients; Fair Health: New York, NY, USA, 2020.

5. Scottish Government. Impacts of COVID-19 on Equality in Scotland. 2020. Available online: https://www.gov.scot/publications / the-impacts-of-covid-19-on-equality-in-scotland/ (accessed on 9 February 2021).

6. Chart of the Week: COVID-19 Continues to Disproportionately Affect the Health and Wellbeing of Disabled People. Available online: https:/ / www.nuffieldtrust.org.uk/ resource/chart-of-the-week-covid-19-continues-to-disproportionally-affect-healthand-wellbeing-of-disabled-people (accessed on 29 April 2021).

7. The Scottish Commission for People with Learning Disabilities (2020). The Impact of Coronavirus on People with Learning Disabilities, Their Parents, Carers, and Supporters. Available online: https://www.scld.org.uk/wp-content/uploads/2020/06/ SCLD-Coronavirus-Report-Easy-read-Designed-FINAL.pdf (accessed on 29 April 2021).

8. Accessible Information Needs to Be a Priority during and beyond the Coronavirus Pandemic. Available online: https://www. birmingham.ac.uk/news/thebirminghambrief/items/2020/12/accessible-information-needs-to-be-a-priority-clarity.aspx (accessed on 29 April 2021).

9. The Scottish Government. The Keys to Life: Improving Quality of Life for People with Learning Disabilities; The Scottish Government: Edinburgh, UK, 2013.

10. United Nations. Convention on the Rights of Persons with Disabilities and Optional Protocol. 2006. Available online: https: //www.un.org/disabilities/documents/convention/convoptprot-e.pdf (accessed on 19 April 2021).

11. NHS England. Accessible Information: Specification v1.1; NHS England: London, UK, 2017.

12. NHS Scotland. Recover, restore, renew. In Chief Medical Officer for Scotland; Annual Report 2020-2021; Scottish Government: Edinburgh, UK, 2021.

13. Royal College of Speech and Language Therapists. Adults with Learning Disabilities (ALD)—Position Paper; RCSLT: London, UK, 2010.

14. Sutherland, R.J.; Isherwood, T. The evidence for easy-read for people with intellectual disabilities: A systematic literature review. J. Policy Pract. Intellect. Disabil. 2016, 13, 297-310. [CrossRef]

15. Hurtado, B.; Jones, L.; Burniston, F. Is easy-read information really easier to read? J. Intellect. Disabil. Res. 2014, 58, 822-829. [CrossRef] [PubMed]

16. Walmsley, J. Access in mind: A review of approaches to accessible information for people with learning disabilities. In Understanding and Promoting Access for People with Learning Difficulties; Seale, J., Nind, M., Eds.; Taylor and Francis: Abingdon, UK, 2010; pp. 23-44.

17. Walmsley, J. Commentary on 'Enabling access to information by people with learning disabilities. Tizard Learn. Disabil. Rev. 2013, 18, 16-19. [CrossRef]

18. Buell, S.; Langdon, P.E.; Pounds, G.; Bunning, K. An open randomized controlled trial of the effects of linguistic simplification and mediation on the comprehension of "easy read" text by people with intellectual disabilities. J. Appl. Res. Intellect. Disabil. 2020, 33, 219-231. [CrossRef]

19. Chinn, D. Talking to producers of Easy Read health information for people with intellectual disability: Production practices, textual features, and imagined audiences. J. Intellect. Dev. Disabil. 2019, 44, 410-420. [CrossRef]

20. Mander, C. First-hand experience of accessible information. Tizard Learn. Disabil. Rev. 2015, 20, 80-87. [CrossRef]

21. Oldreive, W.; Waight, M. Enabling access to information by people with learning disabilities. Tizard Learn. Disabil. Rev. 2013, 18, 5-15. [CrossRef]

22. Hyland-Wood, B.; Gardner, J.; Leask, J.; Ecker, U.K. Toward effective government communication strategies in the era of COVID-19. Humanit. Soc. Sci. Commun. 2021, 8, 1-11. [CrossRef]

23. Jarrett, D.; McGregor, S.A.; Terras, M.M. The Use of Accessible Information in the Healthcare of People with Learning Disabilities; Final Project Report Submitted NHS Ayrshire \& Arran: Ayr, Scotland, 2015.

24. Terras, M.M.; McGregor, S.A.; Jarrett, D. Examining the Use of Accessible Information in the Health Care of Adults with Learning Disabilities: A Mixed-Method Approach; SAGE Publications Ltd.: London, UK, 2017.

25. NHS Commissioning for Carers. Available online: https://www.eng;andnhs.uk/commissioning/comm-carers (accessed on 7 June 2021).

26. Doherty, A.J.; Atherton, H.; Boland, P.; Hastings, R.; Hives, L.; Hood, K.; James-Jenkinson, L.; Leavey, R.; Randell, E.; Reed, J.; et al. Barriers and facilitators to primary health care for people with intellectual disabilities and/or autism: An integrative review. BJGP Open 2020. [CrossRef]

27. Gale, N.K.; Heath, G.; Cameron, E.; Rashid, S.; Redwood, S. Using the framework method for the analysis of qualitative data in multi-disciplinary health research. BMC Med. Res. Methodol. 2013, 13, 117. [CrossRef] [PubMed] 
28. Virtual Inclusive Partners and NHS Ayrshire and Arran Health Promotion. Beating the Blues; NHS Ayrshire and Arran: Ayr, Scotland, 2011.

29. Phillips, L. Learning disabilities: Making reasonable adjustments in hospital. Nurs. Times 2019, 115, 38-42.

30. Waight, M.; Oldreive, W. Investigating accessible information formats with people who have learning disabilities. Learn. Disabil. Pract. 2020. [CrossRef]

31. Terras, M.M.; Hendry, G.; Jarret, D. The challenges of safety and community integration for vulnerable individuals. Safety 2019, 5, 85. [CrossRef]

32. MENCAP. Am I Making Myself Clear? Mencap's Guidelines for Accessible Writing. 2002. Available online: https:/ /www.mencap. org.uk/node/ 6040 (accessed on 27 April 2021).

33. Willner, P.; Rose, J.; Stenfert Kroese, B.; Murphy, G.H.; Langdon, P.E.; Clifford, C.; Cooper, V. Effect of the COVID-19 pandemic on the mental health of carers of people with intellectual disabilities. J. Appl. Res. Intellect. Disabil. 2020, 33, 1523-1533. [CrossRef]

34. Coutts, P. COVID-19 and Communities Listening Project: A Shared Response; Carnegie UK Trust: Dunfermline, UK, 2020.

35. Fog Heen, A.; Montori, V.M. Achieving care: Promoting alternatives to industrial healthcare. Swiss Med. Wkly. 2019, 149, w20115. [CrossRef]

36. Chinn, D.; Pelletier, C. Deconstructing the co-production ideal: Dilemmas of knowledge and representation in a co-design project with people with intellectual disabilities. J. Intellect. Dev. Disabil. 2020, 45, 326-336. [CrossRef]

37. Wherton, J.; Greenhalgh, T. Evaluation of the Near Me Video Consulting Service in Scotland during COVID-19, 2020; Scottish Government: Edinburgh, UK, 2020.

38. Harris, J. The use, role and application of advanced technology in the lives of disabled people in the UK. Disabil. Soc. 2010, 25, 427-439. [CrossRef]

39. Chiner, E.; Gómez-Puerta, M.; Cardona-Moltó, M.C. Internet use, risks and online behaviour: The view of internet users with intellectual disabilities and their caregivers. Br. J. Learn. Disabil. 2017, 45, 190-197. [CrossRef]

40. Glencross, S.; Mason, J.; Katsikitis, M.; Greenwood, K.M. Internet use by people with intellectual disability: Exploring digital inequality-A systematic review. Cyberpsychol. Behav. Soc. Netw. 2021. [CrossRef]

41. Heitplatz, V.N.; Bühler, C.; Hastall, M.R. Usage of digital media by people with intellectual disabilities: Contrasting individuals' and formal caregivers' perspectives. J. Intellect. Disabil. 2021. [CrossRef] [PubMed]

42. Johansson, S.; Gulliksen, J.; Gustavsson, C. Disability digital divide: The use of the internet, smartphones, computers and tablets among people with disabilities in Sweden. Univers. Access Inf. Soc. 2021, 20, 105-120. [CrossRef]

43. Sweller, J. Cognitive load during problem solving: Effects on learning. Cogn. Sci. 1988, 12, 257-285. [CrossRef]

44. Castro Alonso, J.; de Koning, B.B. Latest Trends to Optimize Computer-Based Learning: Guidelines from Cognitive Load Theory; Elsevier: Amsterdam, The Netherlands, 2020.

45. Sweller, J.; van Merri€enboer, J.J.G.; Paas, F. Cognitive architecture and instructional design: 20 years later. Educ. Psychol. Rev. 2019, 31, 261-292. [CrossRef]

46. Mental Health Foundation. Coronavirus: The Divergence of Mental Health Experiences during the Pandemic; Mental Health Foundation: London, UK, 2020. 